\title{
Stable Configurations of Charged Sedimenting Particles
}

\author{
C. I. Trombley and M. L. Ekiel-Jeżewska* \\ Institute of Fundamental Technological Research, Polish Academy of Sciences, Pawińskiego 5b, 02-106 Warsaw, Poland
}

(Received 5 October 2018; revised manuscript received 27 November 2018; published 21 December 2018; corrected 21 July 2021)

\begin{abstract}
The qualitative behavior of charged particles in a vacuum is given by Earnshaw's theorem, which states that there is no steady configuration of charged particles in a vacuum that is asymptotically stable to perturbations. In a viscous fluid, examples of stationary configurations of sedimenting uncharged particles are known, but they are unstable or neutrally stable - they are not attractors. In this Letter, it is shown by example that two charged particles settling in a fluid may have a configuration that is asymptotically stable to perturbations for a wide range of charges, radii, and densities. The existence of such "bound states" is essential from a fundamental point of view and it can be significant for dilute charged particulate systems in various biological, medical, and industrial contexts.
\end{abstract}

DOI: 10.1103/PhysRevLett.121.254502

Earnshaw's theorem gives fundamental insights into the stability of charged systems. Introduced in Ref. [1], the theorem states that there is no stable equilibrium of charged particles distributed in a vacuum without a boundary. An informal reading is that electrostatic interactions are inherently destabilizing and one must add, e.g., boundaries or stabilizing forces [2]. Historically, Earnshaw's theorem informed the development of models of the stability of matter and studies of qualitative features of charged systems [2,3]. Finding the stable configurations allowed by Earnshaw's theorem when a spherical boundary is imposed-the "Thompson problem"-is an active field [4]. Earnshaw's theorem underpins classical models of Wigner crystallization (for instance, see Ref. [5]). It even allows one to find quantitative limits on parameters for stable classical models of complex molecules [6]. In this Letter, we show that the presence of an unbounded electrically neutral fluid can stabilize systems of charged microparticles.

At micro and nano scales, both active "agents" and passive objects, whether of biological [7-9] or inorganic materials $[10,11]$, and naturally or artificially made, have been modeled theoretically as particles in a fluid. In general, such particles can have complex shapes and be deformable. Their rich dynamics have been extensively investigated [12-19]. The development of microfluidics, Lab-On-Chip technologies [20], and advances in medicine and the design of innovative materials and devices-e.g., to

Published by the American Physical Society under the terms of the Creative Commons Attribution 4.0 International license. Further distribution of this work must maintain attribution to the author(s) and the published article's title, journal citation, and DOI. carry drugs [21] or treat wastewater [22]—depends on this research.

The concept of a noninertial "Stokes flow," introduced in Ref. [23], holds a central place in the theory of the dynamics of micro and nano particles $[24,25]$. In particular, Stokes equations are widely used to determine the influence of a viscous fluid on the dynamics of particles experiencing external forces, such as gravity or in a centrifuge [14,26-30]. For a single particle, Stokes flow is an appropriate model when the particle has reached its terminal velocity, its so-called Stokes velocity. The terminal velocity is reached swiftly at a microscale. In systems of microparticles in a Stokes flow, the velocity of each particle is a linear combination of the forces on every particle. The coefficients of this combination depend on positions of all the particles.

The goal of this Letter is to find asymptotically stable configurations of two sedimenting charged particles. The existence of such "attractive states" (configurations such that if the particles were disturbed from this configuration then they would tend to return) may be of a great significance for sedimenting suspensions that exhibit electrostatic interparticle interactions.

First, we briefly outline known results for uncharged particles. Owing to reversibility of Stokes equations, identical spherical sedimenting particles can form steady configurations, such as, e.g., horizontal regular polygons made of arbitrary numbers of particles [31]. More simply, any arrangement of two identical particles in free space is steady. However, these steady configurations are, at most, neutrally stable, and therefore are not attractive.

The more interesting case of two spherical uncharged sedimenting particles with different radii and densities was examined in the seminal paper [32]. If the particles are far enough from each other that their interaction can be neglected, then particle $A$ with a larger Stokes velocity 
will fall faster than particle $B$ with a smaller Stokes velocity. Intuition may suggest that if particle $A$ is above particle $B$ with their centers in a vertical line, then they will tend to approach each other no matter what their distance. Counterintuitively, it was found that, in a certain range of parameters, the particles do not tend to touch each other (in an infinite time) but instead "capture" each other at a distance a bit larger than the sum of their radii [32]. Even more surprisingly, particle $A$ can move slower than $B$ if the interparticle distance is smaller than its steady value. In this uncharged system, vertical steady configurations are stable against vertical, but unstable with respect to horizontal, perturbations.

The main idea of this Letter is to introduce charge to such a system, to find a steady vertical configuration and check if electrostatic attraction between the particles will cause them to come back to the steady configuration if perturbed. In the following, we will show that indeed this is the casewe discover stable configurations. Counterintuitively to Earnshaw's theorem in vacuum, electrostatic interactions between charged particles in fluids can play a stabilizing role.

We now introduce a model of two charged, spherical particles settling under gravity in a fluid of dynamic viscosity $\mu$. We assume that Brownian motion, fluid compressibility, and inertia are irrelevant, and we describe the fluid flow by the Stokes equations [24,25]. Thus, the external forces on the particles are in balance with the fluid resistance forces, and therefore the dynamics of particles is described by a system of first order differential equations.

We denote particle radii by $a_{1}$ and $a_{2}$. Let $M_{1}$ and $M_{2}$ represent the mass of particle 1 and 2 . The reduced density of each particle is the difference between its density and the density $\rho$ of the fluid. Similarly, $m_{1}=M_{1}-\frac{4}{3} \pi a_{1}^{3} \rho$ and $m_{2}=M_{2}-\frac{4}{3} \pi a_{2}^{3} \rho$ are the reduced masses. We assume $m_{2}>0$ with other cases covered in the Supplemental Material [33]. Let $\mathbf{r}_{1}$ and $\mathbf{r}_{2}$ be the positions of the centers of particle 1 and 2. Then the relative position is $\mathbf{d}=\mathbf{r}_{2}-\mathbf{r}_{1}$. We choose a coordinate system so that the particle centers and the direction of gravity are in the plane $y=0$ and $\hat{\mathbf{z}}$ is a unit vector pointing antiparallel to the constant gravitational field $\boldsymbol{g}$. We can now write the superposition of electrostatic and gravitational forces on the particles 1 and 2

$$
\begin{aligned}
& \mathbf{f}_{1}=-k q_{1} q_{2} \frac{\mathbf{d}}{|\mathbf{d}|^{3}}-m_{1} g \hat{\mathbf{z}} \\
& \mathbf{f}_{2}=k q_{1} q_{2} \frac{\mathbf{d}}{|\mathbf{d}|^{3}}-m_{2} g \hat{\mathbf{z}},
\end{aligned}
$$

where $k$ is Coulomb's constant, $q_{1}$ and $q_{2}$ are the charges on particles 1 and $2,|\mathbf{v}|$ is the length of any vector $\mathbf{v}$, and $g=|\boldsymbol{g}|$. Assuming pointlike charges, we consistently take a point particle approximation for the interaction with the fluid [25,34], and we obtain the following system of ordinary differential equations (ODEs):

$$
\begin{aligned}
& \dot{\mathbf{r}}_{1}=\frac{1}{8 \pi \mu} \boldsymbol{G} \cdot \mathbf{f}_{2}+\frac{1}{6 \pi \mu a_{1}} \mathbf{f}_{1} \\
& \dot{\mathbf{r}}_{2}=\frac{1}{8 \pi \mu} \boldsymbol{G} \cdot \mathbf{f}_{1}+\frac{1}{6 \pi \mu a_{2}} \mathbf{f}_{2},
\end{aligned}
$$

where $G_{i j}=\delta_{i j} /|\mathbf{d}|+d_{i} d_{j} /|\mathbf{d}|^{3}$ is the Green tensor for the Stokes equations in an unbounded fluid [25]. The total velocity of each particle has a mutual part that depends on the force on the other particle and a self-part that depends on the force on the particle itself. Notice that it is necessary to take into account the particle radii in the self-terms. Because of the translational invariance of the system $\boldsymbol{G}$ depends only on the relative position $\mathbf{d}$. We are interested in the relative motion, which satisfies the following ODEs

$$
\begin{aligned}
\dot{\mathbf{d}}= & \frac{1}{8 \pi \mu}\left(-\frac{2 k q_{1} q_{2}}{|\mathbf{d}|^{3}} \boldsymbol{G} \cdot \mathbf{d}+\left(m_{2}-m_{1}\right) g \boldsymbol{G} \cdot \hat{\mathbf{z}}\right) \\
& -\frac{1}{6 \pi \mu}\left(-\frac{k q_{1} q_{2}}{|\mathbf{d}|^{3}}\left(\frac{1}{a_{1}}+\frac{1}{a_{2}}\right) \mathbf{d}+\left(\frac{m_{2}}{a_{2}}-\frac{m_{1}}{a_{1}}\right) g \hat{\mathbf{z}}\right) .
\end{aligned}
$$

Before we examine the properties of Eq. (5), we describe physical properties of the system using nondimensional parameters, which are independent of each other and constant during particle motion

$$
\gamma=\frac{a_{1}}{a_{2}}, \quad \delta=\frac{m_{1}}{m_{2}}, \quad \beta=-\frac{k q_{1} q_{2}}{L^{2} m_{2} g},
$$

so that $\gamma$ is the ratio of particle radii, $\delta$ is the ratio of reduced particle masses, and $\beta$ is the ratio of characteristic Coulomb force $F_{e}=-k q_{1} q_{2} / L^{2}$ to the characteristic gravitational force $F_{g}=m_{2} g$. The sign of $F_{e}$ is chosen to be positive when the charges attract each other. There are some physically interesting functions of these parameters. For instance, the ratio of reduced densities is $\delta / \gamma^{3}$ and the ratio of Stokes velocities is $\delta / \gamma$.

We now choose the units

$$
L=a_{1}+a_{2}, \quad V=\frac{m_{2} g}{6 \pi \mu L}
$$

where $L$ - the characteristic length-is the distance the particle centers would have if the particle surfaces were in contact, and $V$ is a characteristic velocity. These scales define a characteristic timescale $T=L / V$. Notice that changing the viscosity $\mu$ modifies only the velocity and timescales. The nondimensional parameters [Eq. (6)] are invariant. In the Stokes regime, changes in viscosity do not alter the paths on which the particles move but only the rate at which they move on said paths [24,25]. 
Finally, we nondimensionalize the relative position

$$
\alpha=\frac{\mathbf{d}}{L}
$$

so that, if the particle surfaces were in contact, $|\boldsymbol{\alpha}|=1$. We can now write Eq. (5) involving only the nondimensional ratios

$$
\begin{aligned}
\dot{\boldsymbol{\alpha}}= & \frac{3}{2|\boldsymbol{\alpha}|^{3}} \beta \mathcal{G} \cdot \boldsymbol{\alpha}+\frac{3}{4}(1-\delta) \mathcal{G} \cdot \hat{\mathbf{z}}-\beta \frac{(1+\gamma)^{2}}{\gamma|\boldsymbol{\alpha}|^{3}} \boldsymbol{\alpha} \\
& -\frac{(\gamma-\delta)(1+\gamma)}{\gamma} \hat{\mathbf{z}},
\end{aligned}
$$

where $\mathcal{G}_{i j}=\delta_{i j} /|\boldsymbol{\alpha}|+\alpha_{i} \alpha_{j} /|\boldsymbol{\alpha}|^{3}$ and from now on the dot denotes derivative with respect to nondimensional time ratio $t / T$.

We now analyze Eq. (9) and discover a class of vertical configurations that are stable to any perturbation.

We denote a nondimensional stationary configuration by $\alpha^{*}=\alpha^{*} \hat{\mathbf{z}}$, with $\alpha^{*}>0$. Our convention is then to assign label 2 to the particle with larger $\hat{z}$ component in the steady state. To examine the stability of such a configuration, we investigate how the system evolves if we have a first order perturbation $\epsilon$ in the direction perpendicular to gravity and a positive component $\alpha$ in the $\hat{z}$ direction (not necessarily close to $\alpha^{*}$ ). If $\boldsymbol{\alpha}=\epsilon \hat{\mathbf{x}}+\alpha_{z} \hat{\mathbf{z}}$ and we neglect second and higher order terms in $\epsilon$ then $\alpha=|\boldsymbol{\alpha}|=\sqrt{\alpha_{z}^{2}+\epsilon^{2}} \approx \alpha_{z}$. With this, Eq. (9) becomes

$$
\begin{aligned}
& \dot{\epsilon}=g(\alpha) \epsilon \\
& \dot{\alpha}=f(\alpha)
\end{aligned}
$$

where

$f(\alpha)=\frac{6 \gamma \beta-2 \beta(1+\gamma)^{2} \alpha+3 \gamma(1-\delta) \alpha^{2}-2(\gamma-\delta)(1+\gamma) \alpha^{3}}{2 \gamma \alpha^{3}}$

$g(\alpha)=\frac{12 \gamma \beta-4(1+\gamma)^{2} \beta \alpha+3 \gamma(1-\delta) \alpha^{2}}{4 \gamma \alpha^{4}}$.

In the numerator of $f$ the four terms are, consecutively, the mutual and self-parts of velocity arising from electrostatic forces, and the mutual and self-parts of velocity arising from the gravitational forces. This is similar for $g$, except that there is no self-part of horizontal velocity arising from vertical gravitational force.

For any system of differential equations of the form (10) and (11), if $g$ and $f$ are continuous, then the condition for $\alpha^{*}=\alpha^{*} \hat{\mathbf{z}}$ to be an steady state is

$$
f\left(\alpha^{*}\right)=0
$$

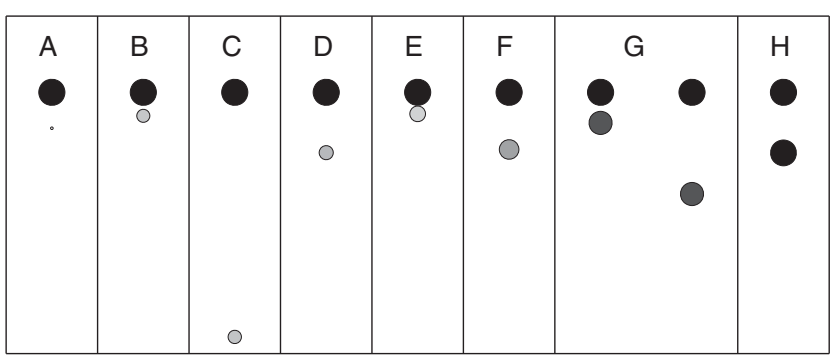

FIG. 1. Examples $A-H$ illustrate stable stationary configurations of charged particles settling under gravity in a Stokes flow for the parameter values listed in Table I. Gravity points down.

If $f$ is continuously differentiable and $g$ is continuous in an open neighborhood of a steady state $\alpha^{*}$, then $\boldsymbol{\alpha}^{*}$ is stable if and only if

$$
\begin{aligned}
g\left(\alpha^{*}\right) & <0 \\
f^{\prime}\left(\alpha^{*}\right) & <0 .
\end{aligned}
$$

A proof that Eqs. (14)-(16) are necessary and sufficient for local asymptotic stability [35] is given in section II of the Supplemental Material [33].

Finally, we impose the feasibility condition

$$
1<\alpha^{*}
$$

in order to rule out ghostlike overlapping particles.

We now demonstrate that there exist solutions to Eqs. (14)-(17). We provide examples of stable stationary feasible configurations in Fig. 1 with the parameters in Table I. In Fig. 1, the density of particle 2 is held constant and painted black, while brighter colors are used to represent denser particles. Similarly, radius $a_{2}$ of the upper particle is taken to be the same across columns and the radius $a_{1}$ of the lower particle is drawn to scale.

In case $A$, small $\delta$ and $\gamma$ are chosen. This corresponds to the higher particle being much larger and more massive than the lower particle. Case $B$ shows that stability is possible when $\delta=\gamma$. This corresponds to particles that

TABLE I. Positions and parameters of the stable stationary configurations shown in Fig. 1.

\begin{tabular}{lllllll}
\hline \hline & \multicolumn{1}{c}{$\alpha^{*}$} & \multicolumn{1}{c}{$\beta$} & \multicolumn{1}{c}{$\delta$} & \multicolumn{1}{c}{$\gamma$} & \multicolumn{1}{c}{$\delta / \gamma$} & \multicolumn{1}{c}{$\delta / \gamma^{3}$} \\
\hline$A$ & 2.5 & $0.160 \ldots$ & 0.075 & 0.1 & 0.75 & 75 \\
$B$ & 1.2 & 0.45 & 0.5 & 0.5 & 1 & 4 \\
$C$ & 12.4 & $1.12 \ldots$ & 0.5 & 0.51 & $0.980 \ldots$ & $3.76 \ldots$ \\
$D$ & 3 & $0.360 \ldots$ & 0.5 & 0.54 & $0.925 \ldots$ & $3.17 \ldots$ \\
$E$ & 1.03 & $0.930 \ldots$ & 1.1 & 0.6 & $1.83 \ldots$ & $5.09 \ldots$ \\
$F$ & 2.5 & $0.212 \ldots$ & 0.72 & 0.75 & $0.960 \ldots$ & $1.70 \ldots$ \\
$G$ & $1.24 \ldots$ & 0.125 & 0.875 & 0.885 & $0.988 \ldots$ & $1.26 \ldots$ \\
& $4.13 \ldots$ & & & & & \\
$H$ & $2.33 \ldots$ & $0.00997 \ldots$ & 0.986 & 0.988 & $0.997 \ldots$ & $1.02 \ldots$ \\
\hline \hline
\end{tabular}


have identical Stokes velocities. Next we look at cases $C$ and $D$ where the separation distance $\alpha^{*}$ is large. Case $E$ gives an example where $\delta / \gamma>1$, so that the lower particle has a greater Stokes velocity than the upper particle. Case $G$ illustrates that, for the same parameters, two distinct stable stationary configurations can exist. In case $H, \gamma \approx 1, \delta \approx 1$, and $\beta \approx 0$, showing that there are stable stationary configurations very close to the classic case of two identical uncharged particles.

Now that we know that the solution set is nonempty, we investigate the range of parameters consistent with a stable feasible steady configuration. The range will come directly from the necessary and sufficient conditions [Eqs. (14)-(17)]. The physical implications of these bounds will also be discussed.

We start with the ratio of characteristic electrostatic to characteristic gravitational force $\beta$. By manipulating the conditions [Eqs. (14)-(17)], one can see

$$
\frac{3 \beta}{\alpha^{* 3}}=3 f\left(\alpha^{*}\right)-\alpha^{*}\left[f^{\prime}\left(\alpha^{*}\right)+2 g\left(\alpha^{*}\right)\right]>0 .
$$

Therefore, if a solution exists, then $\beta>0$. This means that the particles must attract each other in order for the system to be stable, in agreement with our predictions that motivated this Letter. This is also important because it allows for stable systems that have a zero net charge, $q_{1}+q_{2}=0$.

Next, we show that the ratio of reduced masses $\delta>0$. We use that $f\left(\alpha^{*}\right)-\alpha^{*} g\left(\alpha^{*}\right)>0$ to solve for a bound on $\delta$ to get

$$
\delta>\frac{3 \gamma-4 \gamma(1+\gamma) \alpha^{*}}{3 \gamma-4(1+\gamma) \alpha^{*}}>0,
$$

because the denominator and numerator are both necessarily negative if $\gamma>0$ and $\alpha^{*}>1$. This demonstrates that if $m_{2}>0$, then $m_{1}>0$. In the Supplemental Material [33], we extend this to show that stable doublets can exist only in the $m_{2}>0$ and $m_{1}>0$ case and the symmetric case when buoyancy dominates over gravity $m_{2}<0 \& m_{1}<0$.

Moreover, the upper particle must have a larger radius than the lower particle

$$
\gamma<1 \text {. }
$$

The demonstration is somewhat tedious, so it is given in the Supplemental Material [33].

If we divide both sides of Eq. (19) by $\gamma^{3}$, we can use $\gamma<1$ and Eq. (17) to show that the middle term in Eq. (19) will be larger than 1 . Therefore,

$$
\delta / \gamma^{3}>\frac{3-4(1+\gamma) \alpha^{*}}{\left(3 \gamma-4(1+\gamma) \alpha^{*}\right) \gamma^{2}}>1 .
$$

This means that the lower particle has to be more dense than the upper one. This has the interesting implication that
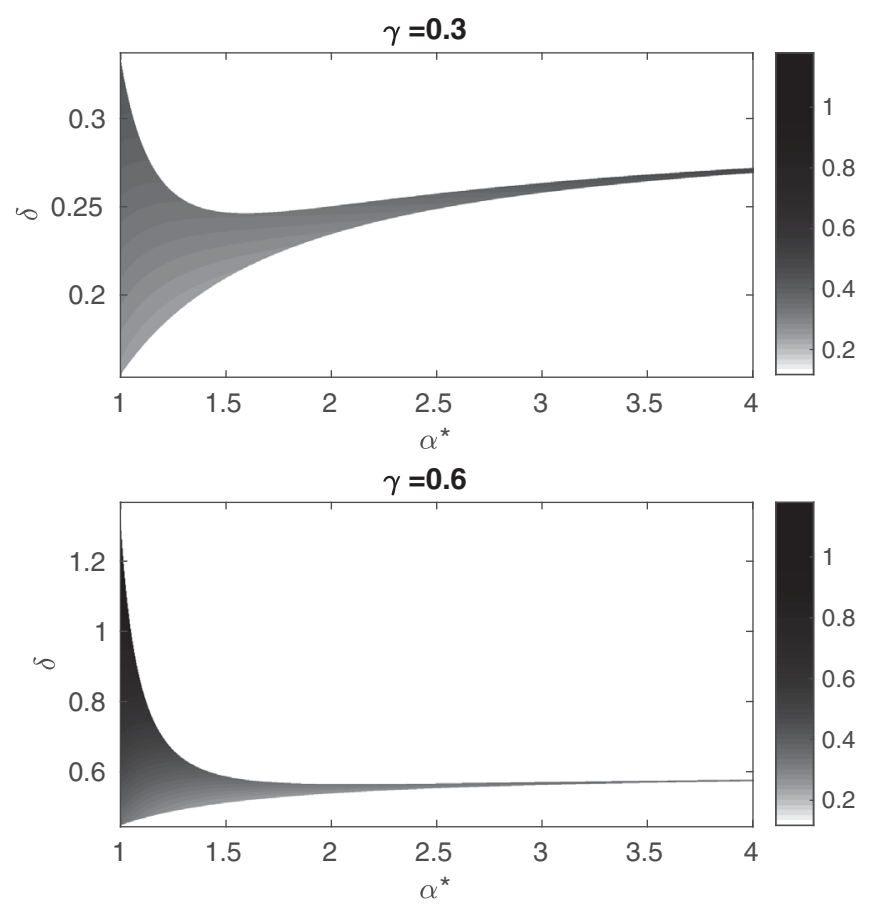

FIG. 2. Regions of stable steady states in parameter space are plotted. Given $\gamma$ and $\delta$, the shade at a point is chosen by characteristic force ratio $\beta$ necessary to stabilize the system at $\alpha^{*}$. If no amount of charge would stabilize a system with the given parameters, the point is left white.

in our model stable doublets only form between particles of different material.

With these bounds in mind, we give Fig. 2 in order to illustrate the way $\alpha^{*}$ and the parameters $\delta, \gamma$, and $\beta$ are interrelated. These plots visually demonstrate that the set of parameters that allow a feasible stable steady state is large. One can also see that there exist stable stationary configurations in the "tail" where $\alpha^{*}$ gets large. Examination of this tail introduces some facts of physical interest. By expanding the relations [Eqs. (14)-(17)] in powers of $1 / \alpha^{*}$, we deduce that, in the tail, the upper particle must have a slightly greater Stokes velocity than the lower one: $1-(\delta / \gamma) \approx 3(1-\gamma) /[4(1+\gamma)]\left(1 / \alpha^{*}\right) \ll 1$. Looking at the ratio of forces, we see that $\beta \approx 3 \gamma(1-\gamma) /$ $\left[4(1+\gamma)^{2}\right] \alpha^{*} \gg 1$ in the tail. This means that in the tail electrostatic interactions are strong relative to gravitational force. This demonstrates how electrostatic forces can stabilize a doublet even when the distances involved are large.

In another limit, we keep $\alpha^{*}$ constant and move values of $\delta$ and $\gamma$ closer and closer to unity. In this limit, the ratio of Stokes velocities $\delta / \gamma$ and relative densities $\delta / \gamma^{3}$ approach 1 - that is, the particles get more similar. As a consequence of Eq. (14), $\beta$ scales down to $\beta \ll 1$. We are seeing therefore that a small charge can be expected to stabilize the system in this limit.

In order to aid physical intuition in interpreting the above results, we will demonstrate the role of charge in stabilizing 
a system of settling particles. For an uncharged system, the analog of Eq. (14) is $3 \gamma(1-\delta)-2(\gamma-\delta)(1+\gamma) \alpha^{*}=0$. The first and second terms are the contributions of gravity to the mutual and self-parts of velocity. The semistability condition - the analog of Eq. (16) — can be combined with the analog of Eq. (14) to get $-2(\gamma-\delta)(1+\gamma)<0$. Therefore the semistability condition entails the ratio of Stokes velocities $\delta / \gamma<1$, so that the self-term tends to bring the particles together. We also have that $\delta<1$; therefore the mutual term must tend to push the particles apart. These contributions to the velocity balance exactly at a certain distance $\alpha^{\prime}$. However, if the particles are perturbed even slightly in the horizontal direction, the story is different. The analog of Eq. (16) is $3 \gamma(1-\delta)<0$, which cannot be satisfied if $\delta<1$. The horizontal velocitywhich contains only this mutual term-is tending to push the particles apart. There is no asymptotically stable $\alpha^{*}$. At $\alpha$ ' the system is "semi-stable"-it is stable only to perturbations in the vertical direction. For example, $\alpha$ ' $\approx 5.21 \ldots$ in the uncharged system $H^{\prime}$, which has the same mass ratio $\delta$ and ratio of radii $\gamma$ as system $H$ given in Table I and illustrated in Fig. 1.

Now consider adding a very slight charge to the system so that $\beta=0.00997 \ldots$, in other words system $H$. In a vertical arrangement, the electrostatic force adds to the self and mutual contributions to velocity without changing their signs, so that the first thing we find is that the stationary configuration of the system contracts to $\alpha^{*} \approx 2.33 \ldots$. Now consider horizontal perturbations. There are two electrostatic contributions to the horizontal motion. A mutual term, $12 \gamma \beta=0.118 \ldots$, which tends to push the particles apart and a self term, $-4(1+\gamma)^{2} \beta \alpha^{*}=-0.367 \ldots$, which tends to bring them back. As before, the gravitational contribution to horizontal velocity, $3 \gamma(1-\delta) \alpha^{2}=0.225 \ldots$, tends to push the particles apart. The restoring term dominates. Therefore, system $H$ is stable.

In this way, we have demonstrated ostensively how settling charged particle systems (even with small charge) can have qualitatively new behavior (local asymptotic stability) absent in their uncharged counterparts. It applies also to semidiluted polydispersed suspensions of microparticles.

The core prediction of the model presented in this Letter is the formation of stable asymmetric doublets. The existence of such doublets is experimentally testable. These doublets are not stable without charge, indicating the novelty of the settling dynamics explored here.

In future work, we will expand the model to include electrostatic screening and precise treatment of hydrodynamic interactions between hard spheres with stick boundary conditions, applying the multipole expansion corrected for lubrication [36]. We will also investigate the influence of charge on essential features of the dynamics, such as existence of periodic motions [32].
This Letter was supported in part by Narodowe Centrum Nauki under Grant No. 2014/15/B/ST8/04359. We acknowledge scientific benefits from COST Action MP1305.

* Corresponding author. mekiel@ippt.pan.pl

[1] S. Earnshaw, Trans. Cambridge Philos. Soc. 7, 97 (1842).

[2] Y. Levin, Physica (Amsterdam) 352A, 43 (2005).

[3] W. Jones, Eur. J. Phys. 1, 85 (1980).

[4] A. Bondarenko, M. Karchevskiy, and L. Kozinkin, J. Phys. Conf. Ser. 643, 012103 (2015).

[5] R. G. Nazmitdinov, A. Puente, M. Cerkaski, and M. Pons, Phys. Rev. E 95, 042603 (2017).

[6] F. Mohammad-Rafiee and R. Golestanian, Phys. Rev. E 69, 061919 (2004).

[7] V. Kantsler and R. E. Goldstein, Phys. Rev. Lett. 108, 038103 (2012).

[8] K. Kang and J. K. G. Dhont, Phys. Rev. Lett. 110, 015901 (2013).

[9] Y. Liu, B. Chakrabarti, D. Saintillan, A. Lindner, and O. du Roure, Proc. Natl. Acad. Sci. U.S.A. 115, 9438 (2018).

[10] A. Perazzo, J. K. Nunes, S. Guido, and H. A. Stone, Proc. Natl. Acad. Sci. U.S.A. 114, E8557 (2017).

[11] S. Pawłowska, P. Nakielski, F. Pierini, I. K. Piechocka, K. Zembrzycki, and T. A. Kowalewski, PLoS One 12, e0187815 (2017).

[12] D. E. Smith, H. P. Babcock, and S. Chu, Science 283, 1724 (1999).

[13] L. E. Becker and M. J. Shelley, Phys. Rev. Lett. 87, 198301 (2001).

[14] M. C. Lagomarsino, I. Pagonabarraga, and C. P. Lowe, Phys. Rev. Lett. 94, 148104 (2005).

[15] Y.-N. Young and M. J. Shelley, Phys. Rev. Lett. 99, 058303 (2007).

[16] M. Harasim, B. Wunderlich, O. Peleg, M. Kröger, and A. R. Bausch, Phys. Rev. Lett. 110, 108302 (2013).

[17] A. Farutin, T. Piasecki, A. M. Słowicka, C. Misbah, E. Wajnryb, and M. L. Ekiel-Jeżewska, Soft Matter 12, 7307 (2016).

[18] M. Gruziel, K. Thyagarajan, G. Dietler, A. Stasiak, M. L. Ekiel-Jeżewska, and P. Szymczak, Phys. Rev. Lett. 121, 127801 (2018).

[19] M. Bukowicki and M. L. Ekiel-Jeżewska, Soft Matter 14, 5786 (2018).

[20] J. K. Nunes, K. Sadlej, J. I. Tam, and H. A. Stone, Lab Chip 12, 2301 (2012).

[21] D. A. Edwards, J. Hanes, G. Caponetti, J. Hrkach, A. Ben-Jebria, M. L. Eskew, J. Mintzes, D. Deaver, N. Lotan, and R. Langer, Science 276, 1868 (1997).

[22] C. A. Coutinho and V. K. Gupta, J. Colloid Interface Sci. 333, 457 (2009).

[23] G. G. Stokes, Trans. Cambridge Philos. Soc. 9, 8 (1851).

[24] G. K. Batchelor, An Introduction to Fluid Dynamics (Cambridge University Press, Cambridge, England, 2000).

[25] S. Kim and S. J. Karrila, Microhydrodynamics: Principles and Selected Applications (Butterworth-Heinemann, Boston, 1991). 
[26] R. E. Caflisch, C. Lim, J. H. Luke, and A. S. Sangani, Phys. Fluids 31, 3175 (1988).

[27] N. Lecoq, F. Feuillebois, N. Anthore, R. Anthore, F. Bostel, and C. Petipas, Phys. Fluids A 5, 3 (1993).

[28] M. L. Ekiel-Jeżewska, Phys. Rev. E 90, 043007 (2014).

[29] M. Bargiel and E. M. Tory, Powder Technol. 264, 519 (2014).

[30] G. Saggiorato, J. Elgeti, R. G. Winkler, and G. Gompper, Soft Matter 11, 7337 (2015).

[31] L. Hocking, J. Fluid Mech. 20, 129 (1964).

[32] E. Wacholder and N. Sather, J. Fluid Mech. 65, 417 (1974).

[33] See Supplemental Material at http://link.aps.org/ supplemental/10.1103/PhysRevLett.121.254502 for theoretical derivations.
[34] M. D. Graham, Microhydrodynamics, Brownian Motion, and Complex Fluids (Cambridge University Press, Cambridge, England, 2018).

[35] P. Glendinning, Stability, Instability and Chaos: An Introduction to the Theory of Nonlinear Differential Equations (Cambridge University Press, Cambridge, England, 1994), p. 37.

[36] B. Cichocki, M. L. Ekiel-Jeżewska, and E. Wajnryb, J. Chem. Phys. 111, 3265 (1999).

Correction: Seven entries in Table I and related text in the third sentence of the second complete paragraph after Eq. (17) contained errors and have been fixed. 